\title{
Thermal Ablation for Papillary Thyroid Microcarcinoma: How Far We Have Come?
}

This article was published in the following Dove Press journal:

Cancer Management and Research

\author{
Yu Min \\ Xing Wang \\ Hang Chen \\ Jialin Chen \\ Ke Xiang \\ Guobing Yin (1)
}

Department of Breast and Thyroid Surgery, The Second Affiliated Hospital of Chongqing Medical University, Chongqing 404100, People's Republic of China
Correspondence: Guobing Yin Department of Breast and Thyroid Surgery, The Second Affiliated Hospital of Chongqing Medical University, No. 74, Linjiang Road, Yuzhong Dist, Chongqing 404100, People's Republic of China Tel +86 I5823267II9

Email yinguobing@cqmu.edu.cn
Purpose: Thermal ablation (TA), as one of the most currently remarkable technologies, has achieved great success in many malignant diseases including but not limited to hepatic and renal carcinoma. In recent years, this technology was gradually introduced to the treatment of papillary thyroid microcarcinoma (PTMC) and even papillary thyroid carcinoma (PTC). Thereby, we summarized the current progress of TA development in the treatment of PTMC. Methods: The latest relevant literature from the PubMed database with keywords "thermal ablation", "papillary thyroid microcarcinoma", "microwave ablation", "radio-frequency ablation", and "laser ablation", among others, were comprehensively reviewed in this article. The follow-up outcomes of patients in these articles were analyzed.

Results: The efficacy and safety of TA including microwave ablation (MWA), laser ablation (LA), and radiofrequency ablation (RFA) in the treatment of PTC and PTC have been intensively studied. Based on existing clinical trials, the relatively long-term follow-up (range, from 6 to 64.2 months) results in MWA, LA, and RFA were satisfactory that tumor volume reduction rate (VRR) reached and even surpass $99 \%$. Compared with routine surgery methods (total thyroidectomy and lobectomy), the incidence rate of complications was relatively lower and the recurrence rate of TA techniques was not statistically significant, whereas the operative time, blood loss, length of hospital stay, and hospital cost were significantly decreased.

Conclusion: TA presents the same satisfactory therapeutic effects but minimal postoperative trauma can significantly improve the patients' quality of life. However, future larger sample, multicenter, and prospective randomized controlled trials are urgently needed to validate the feasibility of TA in dealing with PTMC.

Keywords: thermal ablation, microwave ablation, laser ablation, radiofrequency ablation, papillary thyroid microcarcinoma

\section{Introduction}

In the last few decades, with an increasing prevalence and overdetection rate of differentiated thyroid carcinoma (DTC) on a global scale, ${ }^{1-3}$ the management of which becomes more precise and particularly crucial. In addition, papillary thyroid microcarcinoma (PTMC), defined as the papillary thyroid carcinoma (PTC) with a diameter maximum of $10 \mathrm{~mm}$, accounts for a considerable proportion of the DTC which should also be paid enough attention. ${ }^{4-7}$ To date, thyroid lobectomy and selective central lymph nodule dissection (SCLND) are the predominant treatment for PTMC in China, even though many of these tumor cells never grow, or grow very slowly during the long-term follow-up. ${ }^{8}$ Although great therapeutic progress in the surgery modalities for DTC has been achieved in the last decades, from 
traditional open thyroidectomy to endoscopic thyroidectomy, the complications subsequent to surgery were still observed in recent literature. ${ }^{5,9}$ Therefore, with the growing concerns for the over-detection rate and overtreatment of PTMC, a range of works have tried to explore alternatives for better treatment modalities, including but not limited to active surveillance (AS) ${ }^{4,10,11}$ and thermal ablation (TA). ${ }^{12,13}$

Currently, TA technologies, as one of novel, potentially more acceptable methods with the same treatment effect, have emerged as an interesting candidate which may increase the patients' experience and compliance. For instance, microwave ablation (MWA), as one of the newly invented techniques, can significantly inactivate tumor cells through protein denaturation due to hyperthermia effects. With less operation time and shorter hospitalization stay, ultrasound-guided MWA has been originally applied in clinical practice for benign tumors. ${ }^{14,15}$ Trying to broaden the indications of MWA, theoretical and experimental investigations of this technique for low-risk of PTMC (T1N0M0) have increased tremendously in the past few years. ${ }^{13,16,17}$ Besides MWA, other TA techniques including laser ablation (LA), radiofrequency ablation (RFA) were also gradually introduced to the clinical practice and also achieved the same therapeutic effects. ${ }^{18-22}$ Noticeably, based on the long-term follow-up of patients who underwent these series of techniques, a significant reduction in primary tumor volume, satisfying tumor resolving, and fewer postoperative complications have been documented, compared with routine surgery. ${ }^{12,18,23}$

Therefore, this review intends to summarize the achievements of the past few years in evaluating the efficacy and safety of these TA techniques in dealing with PTMC. Besides, we also quantitatively analyze the specific assessment indicators after the TA operation, which can provide a more intuitive comparison of differences among them.

\section{Thermal Ablation}

Over the past few years, with an important advancement of medical imaging technology and the popularization of individual physical examination, overdetection and earlier staging at diagnosis of thyroid nodules were achieved. Combined with an in-depth exploration of PTC, the characteristics of this type of malignancy, especially unifocal PTMC, were relatively low invasiveness and presented a favorable prognosis. ${ }^{24}$ However, a minority of patients still suffered from lymph node metastasis (LNM) and extra-glandular invasion during the long-term follow-up. ${ }^{6}$ Moreover, once the PTMC was diagnosed, part of these patients was in various degrees of anxiety and upset about the tumor-carrying state. ${ }^{4,25,26}$ Thus, some patients are more willing to accept relatively aggressive interventions instead of AS. Therefore, various TA approaches currently are being introduced into clinical practice as the alternatives for patients with low-risk PTMC. To date, a range of works has determined the advantages and postoperative complications of these TA techniques in dealing with PTMC. Herein, a more detailed review of outcomes in the different study are concluded in the following section.

\section{Microwave Ablation (MWA)}

With the continuous high-frequency electromagnetic wave output, microwaves can cause coagulation necrosis within the tumor by the extensive thermal effect in a short period (Figure 1A). Therefore, MWA, as one of the most frequent TA technologies, was widely used in clinical practice for benign lesions. Moreover, with the increasing exploration of disease indications for thermal ablation, MWA has gradually emerged as an alternative to surgical resection for solid small malignant tumors originated from liver, renal, and lung. ${ }^{27-30}$ Although MWA was a relatively novel technique, a significant decrease in operating time, recovery unit, hospital stay, and sufficient therapeutic effects were determined in recent study works. ${ }^{23,31}$ Regarding benign thyroid nodules including thyroid ${ }^{32}$ and parathyroid adenoma, ${ }^{33}$ MWA has shown an excellent therapeutic effect. Consequently, as MWA technology continues to mature, some researchers began to apply this technology to the treatment of low-risk PTMC (clinical stage of T1N0M0) which can significantly reduce surgical trauma, accelerate postoperative recovery and improve safety (Table 1). ${ }^{13,16,34}$ In the very beginning, based on past extensive experience in the MWA of hepatocellular carcinoma and benign thyroid nodules, Yue et $\mathrm{al}^{17}$ prospectively enrolled a selected small group of patients (21 cases) with PTMC (T1N0M0) to receive MWA. As a result, they calculated, the mean tumor volume has significantly reduced from $89.5 \pm 20.1 \mathrm{~mm}^{3}$ to $8.7 \pm$ $9.3 \mathrm{~mm}^{3}$. However, the tumor's complete disappearance rate was only $19 \%$, which caused the patients' worrying about the effectiveness of MWA. For this reason, three patients chose to undergo the subtotal thyroidectomy and central node dissection within two months after the MWA. Nevertheless, with an encouraging result of a few complications, no recurrence, and metastasis after MWA, they 


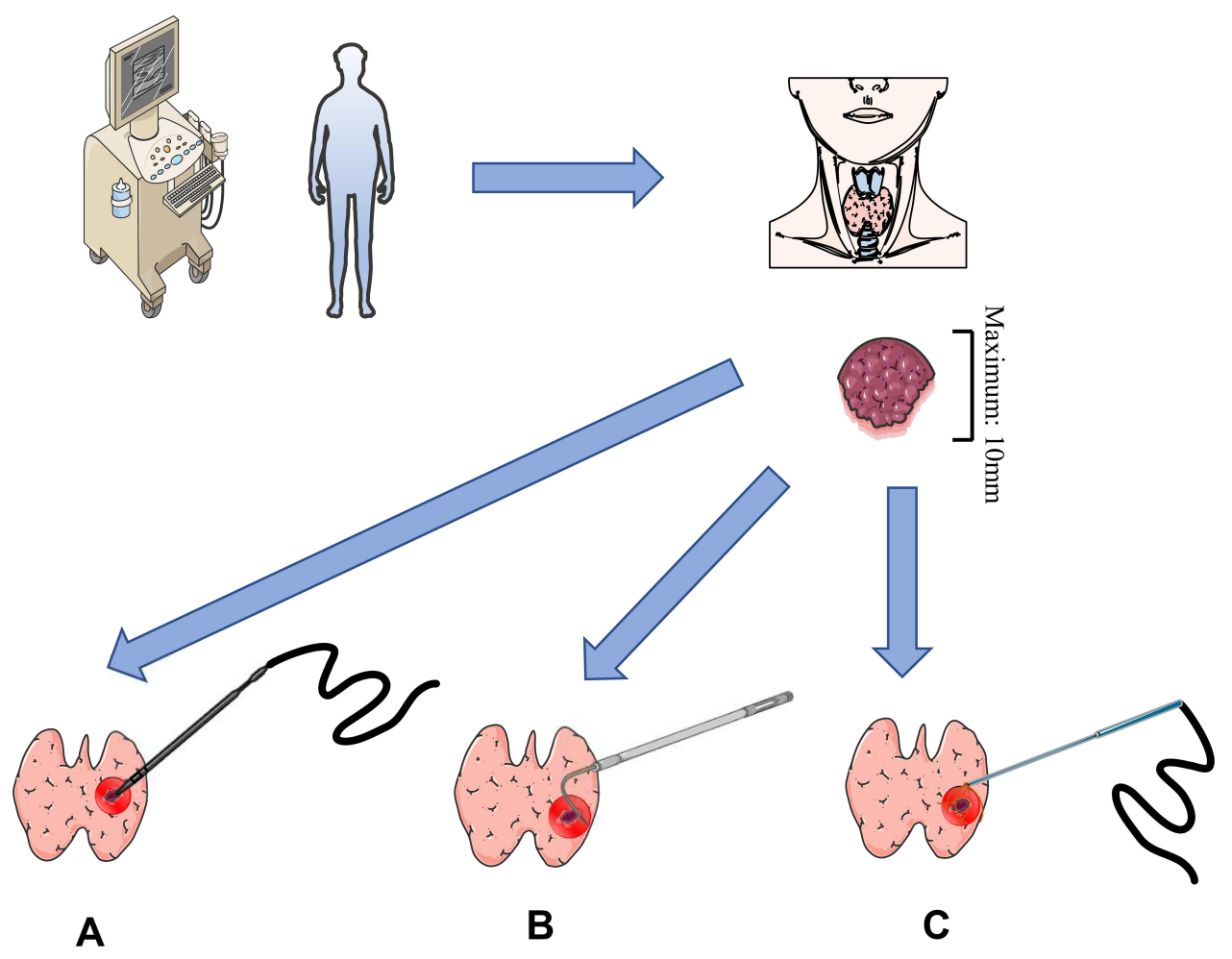

Figure I Different thermal ablation (TA) technologies in dealing with PTMC. (A) microwave ablation (MWA) can induce tumor coagulation necrosis via thermal effects. (B) Laser ablation (LA) can induce heat in the tissue, which in turn leads to coagulative necrosis that destroys tumor cells. (C) Radiofrequency ablation (RFA) uses rapidly alternating radiofrequency current to induce frictional heat around probes placed in tissue, producing cell death by coagulation necrosis.

initially presented a new insight way for the treatment of PTMC. Besides, a series of studies have been performed in this field in the most recent years. With the longer postoperative follow-up and larger study population, the safety and effectiveness of MWA were further consistent determined. To our best of knowledge, as the successional study followed by the previous results (Yue et al), the longest prospective follow-up time was 101 months with an average period of 37.2 months. ${ }^{16}$ Furthermore, in Teng et al original works, the average clinical follow-up duration of forty-one MWA patients reached and even surpassed 60 months which was the longest time so far reported in the literature. ${ }^{13}$ In all cases of these two studies, satisfactory results have been concluded during the long-term follow-up that the volume reduction rate (VRR) achieved to $99.4 \%$ and $99.37 \%$, respectively. The most common complications associated with MWA were burning sensation, hoarseness, and hemorrhage. ${ }^{16,34}$ In these different clinical trials, the power output of the microwave was varied within a range of $20 \mathrm{~W}$ to $40 \mathrm{~W}^{23,31,35}$ The higher the output power and longer procedure time were, the possible increased risk of transient thermal damage to the surrounding tissue may occur. Besides, to ensure the complete tumor resection and prevent marginal recurrence, the extent of ablation was usually $5 \mathrm{~mm}$ beyond the lesions which may also contribute to the impairment of nerves and blood vessels. So far from now, many clinical trials, especially the successional study with long-term follow-up, have concluded that MWA is a promising technology that can be feasible for the treatment of low-risk PTMC. ${ }^{13,16,35}$ These outstanding results may be due to the strict inclusion criteria that the stage was T1aN0M0 and the generally favorable disease prognosis. Interestingly, a majority of observational patients came from Asia, especially China, in literature. And retrospective and prospective study design accounted for most of the trails. Therefore, there is still a lack of high-level clinical evidence from large population-based multiethnic randomized controlled trials (RCTs) to further evaluate the efficacy and safety of the MWA technique.

\section{Laser Ablation (LA)}

As another relevant TA technology (Figure 1B), the fiber during the procedure was connected to a continuous-wave neodymium yttrium-aluminum-garnet (Nd: $\mathrm{YAG}$ ) laser source operating at the wavelength of $1064 \mathrm{~nm}$ with an 
Table I Clinical Outcomes of Thermal Ablation Technologies for PTC or PTMC in Different Studies

\begin{tabular}{|c|c|c|c|c|c|c|c|c|}
\hline $\begin{array}{l}\text { First } \\
\text { Author }\end{array}$ & $\begin{array}{l}\text { Types of } \\
\text { Cancer }\end{array}$ & $\begin{array}{l}\text { Study } \\
\text { Design }\end{array}$ & $\begin{array}{l}\text { No. of } \\
\text { Patient }\end{array}$ & $\begin{array}{l}\text { Mean Follow-Up } \\
\text { Time (Months) }\end{array}$ & $\begin{array}{l}\text { Mean } \\
\text { VRR\% }\end{array}$ & $\begin{array}{l}\text { Complete } \\
\text { Absorption Rate } \\
\%\end{array}$ & $\begin{array}{l}\text { Complication }^{\&} \\
\text { Rate\% (Cases) }\end{array}$ & $\begin{array}{l}\text { Recurrence }{ }^{*} \\
\text { Rate\% (Cases) }\end{array}$ \\
\hline \multicolumn{9}{|l|}{ MWA } \\
\hline$Y_{u e}{ }^{17}$ & PTMC & Pro & 18 & 11.0 & 90.00 & 19.0 & $33.3(6)$ & $0(0)$ \\
\hline $\mathrm{Li}^{23}$ & PTMC & Retro & 46 & 42.0 & 81.33 & 15.2 & $4.3(2)$ & $0(0)$ \\
\hline $\mathrm{Li}^{31}$ & PTMC & Retro & 168 & 25.1 & - & 22.7 & $4.2(7)$ & $4.20(7)$ \\
\hline Teng $^{35}$ & PTMC & Pro & 15 & 36.0 & 98.78 & 95.2 & $6.6(I)$ & $0(0)$ \\
\hline$X u^{54}$ & PTMC & - & 41 & - & - & - & $4.9(2)$ & - \\
\hline Teng $^{34}$ & PTMC & Retro & 185 & 20.7 & 98.65 & 84.5 & $8.6(16)$ & $0(0)$ \\
\hline Teng $^{13}$ & PTMC & Retro & 41 & 60.0 & 99.37 & 97.6 & $4.8(2)$ & $0(0)$ \\
\hline$Y_{u e}{ }^{16}$ & PTMC & Pro & 119 & 37.2 & 99.40 & 78.1 & $10.9(13)$ & $0.88(1)$ \\
\hline Zhou $^{39}$ & PTMC & Retro & 33 & 23.3 & 99.80 & 97.0 & 9.10 (3) & $0(0)$ \\
\hline \multicolumn{9}{|l|}{ LA } \\
\hline Zhou $^{39}$ & PTMC & Retro & 30 & 13.2 & - & 96.7 & $3.30(I)$ & $0(0)$ \\
\hline Zhang $^{54}$ & PTMC & Retro & 64 & 25.7 & 100.00 & 96.9 & - & $1.56(1)$ \\
\hline Zhou $^{18}$ & PTMC & Retro & 36 & 49.2 & 98.38 & 100.0 & $2.80(\mathrm{I})$ & $5.60(2)$ \\
\hline & PTMC & Retro & 37 & 16.5 & - & 32.4 & $2.70(I)$ & $2.70(\mathrm{I})$ \\
\hline Zhou $^{39}$ & PTMC & Retro & 34 & 22.8 & 96.80 & 79.4 & $2.90(\mathrm{I})$ & $0(0)$ \\
\hline \multicolumn{9}{|l|}{ RFA } \\
\hline Zhang $^{46}$ & PTMC & Pro & 92 & 7.8 & 96.00 & 10.2 & $4.30(4)$ & $0(0)$ \\
\hline $\operatorname{Lim}^{45}$ & PTMC & Retro & 133 & - & 100.00 & 91.4 & $3.00(4)$ & $0(0)$ \\
\hline $\mathrm{Kim}^{44}$ & $\begin{array}{l}\text { PTMC/ } \\
\text { PTC }\end{array}$ & Retro & 6 & 48.5 & 98.50 & 66.7 & $0(0)$ & $0(0)$ \\
\hline Ding $^{19}$ & PTMC & Retro & 37 & 6.0 & 99.34 & 97.4 & $0(0)$ & $0(0)$ \\
\hline $\mathrm{Wu}^{48}$ & PTMC & Retro & 198 & 25.9 & 99.80 & 45.6 & 4.50 (9) & $0.51(1)$ \\
\hline $\mathrm{Xiao}^{47}$ & PTC & Retro & 66 & 20.5 & 99.11 & 57.6 & $3.00(2)$ & $4.50(3)$ \\
\hline $\operatorname{Yan}^{19}$ & PTMC & Retro & 414 & 42.2 & 98.81 & 88.4 & $3.86(16)$ & $3.62(15)$ \\
\hline Zhang $^{12}$ & PTMC & Retro & 94 & 64.2 & - & - & $0(0)$ & $1.06(1)$ \\
\hline
\end{tabular}

Notes: ${ }^{\circledR}$ Complication: Complications are defined as hoarseness, choking, coughing, local infection, skin burning, hypothyroidism, hypoparathyroidism, hemorrhage, and hematoma. * Recurrence: Recurrence is defined as lymph node metastasis and new malignant lesion.

Abbreviations: pro, prospective design; retro, retrospective design; MWA, microwave ablation; LA, laser ablation; RFA, radiofrequency ablation; PTMC, papillary thyroid microcarcinoma; PTC, papillary thyroid carcinoma; VRR, volume reduction rate.

output power of 3-4 W. Under the ultrasound guidance, the thermal energy around the fiber head could accurately ablate to the target tumor. LA, like MWA, was also applied to the treatment of early-stage cancers including small hepatocellular carcinoma, small renal cell carcinoma, and even low-risk basal cell carcinoma. ${ }^{29,36-38}$ Approximately one decade ago, Papini et al, for the first time, introduced the LA for the treatment of a solitary PTMC and further determined a satisfactory therapeutic result. ${ }^{22}$ Later on, a range of works, especially those from China, have reached a similar conclusion on the feasibility and safety of this method. Besides, combined with the feature of focusing, LA had less chance of causing collateral damage to surrounding tissues. However, if the laser temperature around the plane-cut optic fiber was consistently high, especially above $110^{\circ} \mathrm{C}$, it could result in tissue carbonization which could delay wound healing in a short followup. ${ }^{39}$ In the contrast, if the temperature increase was not high enough, moderate heating was not able to destroy tumor cells completely but possibly induced the residual tumor tissue growth. This potential risk of LA treatment was recently determined in vitro experiment of hepatocellular carcinoma (HCC) which was via the PI3K/mTOR/ AKT signaling pathway. ${ }^{40}$ Thus, it's worth notice that temperature controlling plays a pivotally important role in a successful operation. Notably, most recently, one retrospective study firstly compared the efficacy and safety between LA and MWA. ${ }^{39}$ Although many between-group 
comparisons were not statistically significant, the MWA group showed a higher volume reduction rate than the LA group (99.8\% vs $96.8 \%$ ) while the latter group had a lower incidence of complications $(2.9 \%$ vs $9.1 \%)$. This phenomenon could be explained by the output power of LA during the procedure was only $3 \mathrm{~W}$ which was far less than MWA needed. Therefore, heat accumulation was relatively lower in the former method.

In the meantime, our knowledge of the effectiveness of LA for low-risk PTMC comes from a limited number of retrospective studies with short postoperative follow-up (range, 18-52 months). While these clinical trials provided us an insightful view of the achievement of LA in tumor volume reduction and accelerated postoperative rehabilitation, some limitations still exist in the wild application of this novel treatment for PTMC. First, there is not enough clinical evidence to guaranteed complete tumor destruction. Second, a few studies are available on comparing the treatment results in LA to other treatment modalities like MWA, RFA, surgery, and even AS. Nonetheless, LA remains to be an alternative therapy with great potential for low-risk PTMC patients who are ineligible or refuse to undergo surgery due to multiple reasons.

\section{Radiofrequency Ablation (RFA)}

RFA relied on the generation of high-frequency alternating electromagnetic energy resulting in thermal damage to the target lesions which was initially used to dealing with supraventricular tachycardias. ${ }^{41,42}$ The high temperature and instantaneous release of energy could ensure the successful application of RFA for the treatment of solid tumors including liver, kidney, and bone cancers, as well as soft-tissue tumors of the breast, head, and neck. ${ }^{43}$ The promising result in the treatment of multiple cancer types indicated that it potentially could be an effective method in the treatment of PTMC (Figure 1C). Under strict inclusion criteria, two retrospective studies from South Korea ${ }^{44,45}$ and one prospective study from China ${ }^{46}$ have yielded consistent promising results that RFA was safe and effective as a reinforcement treatment option for PTMC. Notably, for years, Zhang et al have been evaluating the feasibility and safety of RFA for PTMC and even PTC. ${ }^{12,46-48}$ Upon closer review, they have already retrospectively analyzed clinicopathological data of over 500 PTMC patients in a single-center from January 2013 to December 2017 who elected the RFA treatment. The complication and recurrence results of these patients were in general acceptable $(0-4.5 \%, 0-4.5 \%$, respectively).
Among 414 patients in another study of Yan et $\mathrm{al}^{20}$, a total of 15 patients suffered from local tumor progression after RAF in which 4 patients $(0.97 \%)$ developed lymph nodule metastasis (LNM) and 10 patients $(2.42 \%)$ developed recurrent PTMC. However, in another latest literature $^{12}$ from the same institution, the results seem to be more optimistic: Ninety-four patients were successfully followed-up for at least 5 years which was the longest follow-up period we have known. Among these patients, only one of them developed a new lesion and without other complications or LNM. Based on the excellent result that the previous study achieved, Xiao et $\mathrm{al}^{47}$ further investigated the efficacies of RFA in sixty-six PTC (T1bN0M0) patients. Although the mean VRR reached $99.11 \%$ at the 30 months follow-up, 2 patients (3\%) were found to have malignant cells at the edge of ablation sites and 1 patient (1.5\%) was found to develop LNM during the follow-up. Therefore, at this time, we should remain cautious regarding the application of RFA to clinical routine practice for early-staged (T1aN0M0) PTC patients as a result of occult metastases or lesions. Although there is still a long way to go to further comprehensively evaluate the feasibility of RFA technology when it is used as an initial treatment in dealing with low-risk PTMC, it may be an alternative promising option for those unable to tolerate surgery or refuse surgery.

\section{Comparison with Surgery}

Currently, surgical intervention is still considered to be the first-line treatment of PTC and it can be used as a reference for evaluating the safety and treatment efficacies of other novel techniques, like TA. In dealing with thyroid benign tumors, TA showed to be a good alternative therapy with faster recovery, fewer complications, higher postoperative quality of life, and shorter hospital stay when compared with thyroidectomy. ${ }^{49,50}$ As for thyroid carcinomas, the recurrence and metastasis rate after the operation is pivotal to be calculated in evaluating the therapeutic efficacy of the treatment. However, to date, a majority of clinical trials associated with determining the efficacy and safety of TA were retrospectively designed, lack a control group, and randomization. Only a few comparative studies have been conducted to compare the outcomes of TA and surgical intervention for PTMC patients (Table 2). As $\mathrm{Li}$ et $\mathrm{al}^{23}$ retrospectively reviewed ninety-two patients who underwent the MWA or surgery for PTMC, they determined that none of the patients in two groups suffered from recurrence or LNM 
Table 2 Clinical Outcomes of Thermal Ablation Technologies for PTMC in Comparative Studies

\begin{tabular}{|c|c|c|c|c|c|c|c|c|c|c|c|c|c|c|c|}
\hline \multirow{2}{*}{$\begin{array}{l}\text { First } \\
\text { Author } \\
\mathrm{Li}^{22}\end{array}$} & \multirow{2}{*}{$\begin{array}{l}\text { Design } \\
\\
\text { Retro }\end{array}$} & \multicolumn{2}{|c|}{ Intervention } & \multicolumn{2}{|c|}{$\begin{array}{l}\text { Procedure } \\
\text { Time (Mins) }\end{array}$} & \multicolumn{2}{|l|}{ Cost } & \multicolumn{2}{|c|}{$\begin{array}{l}\text { Mean } \\
\text { Hospital } \\
\text { Stay } \\
\text { (Days) }\end{array}$} & \multicolumn{2}{|c|}{$\begin{array}{l}\text { Mean } \\
\text { Bleeding } \\
\text { Volume } \\
(\mathrm{mL})\end{array}$} & \multicolumn{2}{|c|}{$\begin{array}{l}\text { Complication } \\
\text { Rate\% (No.) }\end{array}$} & \multicolumn{2}{|c|}{$\begin{array}{l}\text { Recurrence } \\
\text { Rate\% (No.) }\end{array}$} \\
\hline & & MWA & Surg & 10.19 & 75.80 & $9996.56^{\#}$ & $15,342.36$ & 1.30 & 7.47 & 1.54 & 33.10 & $\begin{array}{l}4.30 \\
(2)\end{array}$ & $\begin{array}{l}43.50 \\
(20)\end{array}$ & $0(0)$ & $0(0)$ \\
\hline$X u^{50}$ & Pro & MWA & Surg & 25.02 & 78.80 & - & - & 1.77 & 4.18 & 10.32 & 33.12 & $\begin{array}{l}4.90 \\
(2)\end{array}$ & $15.20(7)$ & - & - \\
\hline $\mathrm{Li}^{27}$ & Retro & MWA & Surg & - & - & - & - & - & - & - & - & $\begin{array}{l}4.20 \\
(7)\end{array}$ & $\begin{array}{l}11.90 \\
(17)\end{array}$ & $\begin{array}{l}4.2 \% \\
(7)\end{array}$ & $\begin{array}{l}4.2 \% \\
(6)\end{array}$ \\
\hline Zhang'12 & Retro & RFA & Surg & 7.99 & 62.90 & 1832.00 & $2,355.00$ & 0.00 & 9.35 & 26.10 & 31.60 & $0(0)$ & $3.75(3)$ & $\begin{array}{l}1.1 \% \\
(I)\end{array}$ & $\begin{array}{l}2.5 \% \\
(2)\end{array}$ \\
\hline Zhou $^{18}$ & Retro & LA & Surg & 25.90 & 74.20 & - & - & 0.15 & 2.58 & - & - & $\begin{array}{l}2.80 \\
(I)\end{array}$ & $6.70(3)$ & $\begin{array}{l}5.6 \% \\
(2)\end{array}$ & $\begin{array}{l}6.7 \% \\
(3)\end{array}$ \\
\hline Zhou $^{38}$ & Retro & MWA & LA & 24.00 & 26.90 & - & - & 0.14 & 0.15 & - & - & $\begin{array}{l}9.10 \\
(3)\end{array}$ & $2.90(1)$ & $0(0)$ & $0(0)$ \\
\hline
\end{tabular}

Notes: ${ }^{\# 9996.56: ~ R e n m i n b i ~(R M B) ; ~ 1832: ~ U S A ~ d o l l a r ~(U S D) . ~}$

Abbreviations: Retro, retrospective; Pro, prospective; Surg, surgery.

during the 42-month follow-up. On the contrary, the average cost of MWA and the related fee was significantly lower when compared with the surgery group (9996.5 \pm 586.47 (RMB) vs $15,342.36 \pm 2226.39$ (RMB), $\mathrm{p}<0.001$ ). Although thyroidectomy was a relatively complete procedure in tumor dissection, yet it increased the risk of injury to recurrent laryngeal nerves or parathyroid glands which might result in hoarseness and hypocalcemia related symptoms. As the sample size continues to expand (311 patients), they further proved the effectiveness and safety of MWA in PTMC treatment. Especially, the recurrence rate and 5-year disease-free survival were no statistical significance between MWA and surgery $(4.2 \%$ vs $4.2 \%)$ while the complications rate was much lower in the former group ( $4.2 \%$ vs $11.9 \%, \mathrm{p}<0.001)$. Besides, compared with routine surgery methods (total thyroidectomy and lobectomy), the incidence rate of complications and recurrence of MWA techniques were not statistically significant, whereas the operative time, blood loss, length of hospital stay, hospital cost (RMB) were significantly decreased (Table 2). ${ }^{12,18,31,51}$ Notably, compared with the surgery group, PTMC patients who underwent RFA had lower scores in "problems with scarring" and "less interest in sex" sections of THYCA-QOL (Thyroid Cancer-Specific Quality of Life Questionnaire. Lower the score is, the better quality of life will be). ${ }^{51}$ This divergence could be possibly interpreted by the following two reasons: RFA is a far less invasive operation with almost invisible scars on the neck that can significantly maintain the satisfactory cosmetic result for patients. Besides, it is believed that the thyroid hormone level can influence reproductive hormone levels as well as sexual and reproductive function. ${ }^{52-54}$ Although the efficacies and positive treatment experiences of TA procedures have been determined in recent researches, still randomized controlled studies involving large numbers of patients and longer follow-up periods are required to further confirm this conclusion.

\section{Complications}

Although postoperative complications were significantly decreased in TA when compared with thyroid lobectomy or total thyroidectomy (Table 2), yet there was a small part of patients that developed symptoms related to thermal damage. The most common complications subsequent TA in existing studies were transient hoarseness and burning sensation while the majority of these symptoms could spontaneously be relieved in a short time. Two reasons may be involved in these impairments. On the one hand, the safety of the TA procedure has a close relationship with the primary tumor location. If the lesions are close to the trachea and in the posterior thyroid region adjacent to the capsule, approaching the region where the recurrent laryngeal nerve traverses, there is a relatively high risk of injury to the recurrent laryngeal nerve during the ablation. On the other 
hand, variation in operators' experience or procedural techniques may also contribute to incidental complications like bleeding and neck swelling. In more detail, compared with MWA, the complications after LA were much lower with a rate of 2.7-3.3\% (Table 1, Figure 2B). ${ }^{21,39,55}$ However, the recurrence rate in LA was relatively higher than MWA and RFA (Table 1, Figure 2C). The cause of this phenomenon was not clear but lower power output and the different operating procedures in these two ablation methods might be involved. Besides, MWA had the highest overall incidence of postoperative complications when compared with other TA techniques (Figure 2B). ${ }^{16,34,56}$ Especially in two retrospective studies ${ }^{16,34}$ with larger sample sizes, sixteen in 185 patients and thirteen in 119 patients, respectively, suffered from various degrees of hoarseness, bleeding, and coughing.

In response to these potential risks and side effects, $\mathrm{Li}$ et al put forward that continuous injection spacer fluid (a mixture of $1 \%$ lidocaine and physiological saline) could significantly take away the heat generated during MWA procedures. Moreover, compared with $30 \mathrm{~W}$ or $40 \mathrm{~W}$, a slightly lower power output $(20 \mathrm{~W})$ could make the process of tumor coagulative necrosis more moderate which would be helpful to decrease the thermal-related complications under the premise of ensuring a sufficient effect. Besides, if the nerve damage related to thermal effect was found during the procedure, the direct injection of a cold dextrose solution was recommended as a simple and effective treatment that could result in rapid symptom resolution. ${ }^{57}$ Nonetheless, more comprehensive preoperative evaluation and precision treatment procedures will need to be explored further in the future.

\section{Outcomes}

The general outcomes of different TA methods were encouraging. The outcome indicators mainly included tumor volume, VRR, absorption rate, recurrence, and lymph node or distant metastasis. Recurrence and LNM are of greater concern than postoperative complications in PTMC patients. As it is known to all, TA aims to achieve tumor coagulative necrosis instead of completely dissecting lesions from patients. Thus, there is an existing risk of incomplete ablation and local recurrence. Besides, the incomplete tumor necrosis may further raise patients' anxiety and concerns about the potential recurrence and LNM. ${ }^{17}$ Therefore, compared with the reduction of volume rate and disappearance of the lesion during the follow-up, initial complete ablation is more pivotal because it directly represents a successful operation procedure and low risk of residual in situ after an operation in lesions. For instance, one case report reviewed a multi-center medical database and enrolled twelve patients who used to be treated with thermal ablation to receive surgical therapy. The postoperative pathology reports confirmed the presence of incomplete ablation in all cases and $66.7 \%$ of them had LNM. ${ }^{58}$ Thus, the authors concluded that TA should be recommended with caution as treatment of operable patients with PTC. Among the three types of TA methods, RFA had a relatively lower
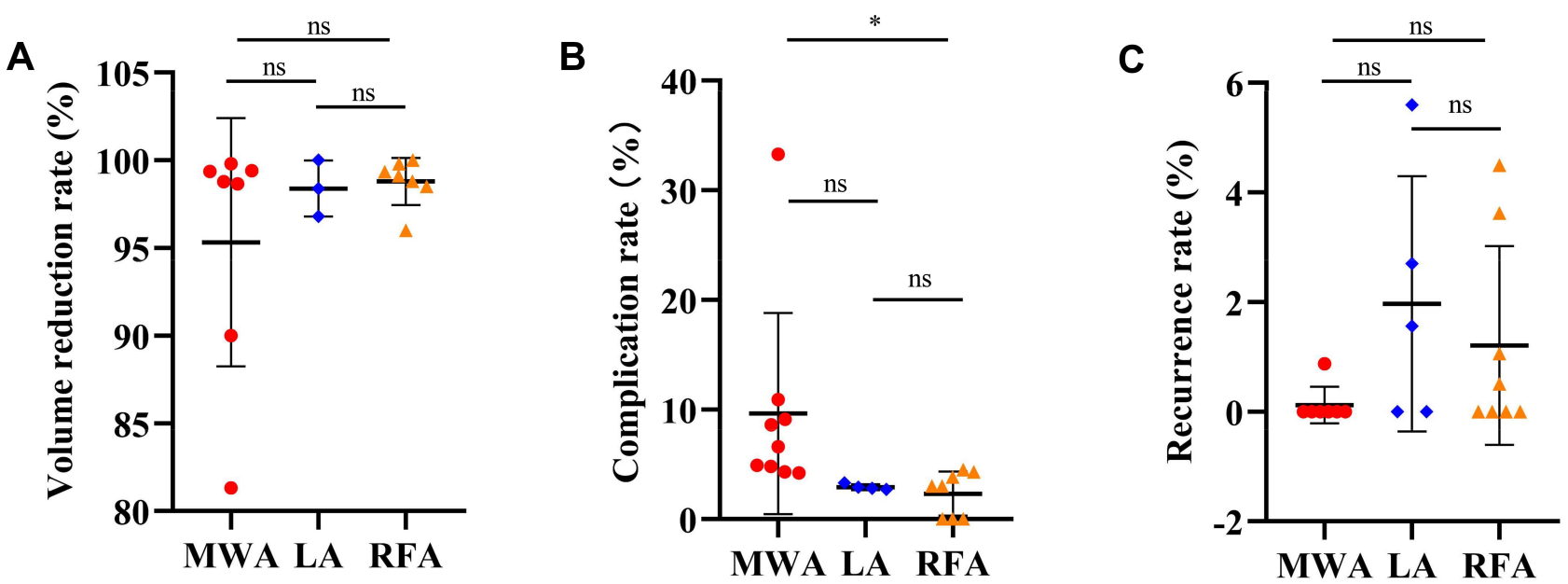

Figure 2 (A) The mean volume reduction rate (VRR) in MWA, LA, and RFA from different retrospective and prospective studies; (B) The complication rate in MWA, LA, and RFA from different retrospective and prospective studies; (C) The recurrence rate in MWA, LA, and RFA from different retrospective and prospective studies; Unpaired $t$-test.

Note: *p-value $<0.05$

Abbreviation: ns, no statistical significance. 
complete ablation rate compared with MWA and LA in recently published research. This phenomenon might be caused by the following reasons: First, a part of the patient's nodule was macro-calcification, the area of which could not be totally ablated. Secondly, compared with RFA used in one vitro study, MWA is rarely affected by the heat-sink effect (local cooling of the thermal process caused by the changes of blood flow) that is thought to contribute to incomplete ablation and the local recurrence after RFA. ${ }^{59}$ Furthermore, compared with satisfied results of VRR, there were great differences in the absorption rate after TA in different trials from $10.2 \%$ to $100 \%$ (Table 1 ). These possible reasons discussed above might also be involved in this divergence.

To increase the accuracy of preoperative localization, a satisfying image can be obtained through the highfrequency color Doppler ultrasound (CDU) which could provide real-time information on the size and location of the malignant nodules. ${ }^{55,60}$ Meanwhile, to reduce the risk of residual lesions and LNM, it's pivotal to access the completeness of TA for treating PTMC in the initial postoperative evaluation. The contrast-enhanced ultrasound (CEUS), as a contrast harmonic imaging technique, was potentially to characterize local lesions by evaluating their micro-vascularization with a second-generation contrast material which was wildly introduced to evaluate the ablative results in renal and hepatic carcinoma. ${ }^{30,60,61}$ Thus, Zhang et $\mathrm{al}^{55}$ suggested that the characteristics of high specificity, sensitivity, and accuracy in CEUS could also be applied to the postoperative evaluation of PTMC that it cannot only access the exact ablation zone but also detect the residual enhancement suspicious lesion.

At present, many studies have determined a low recurrence rate in those PTMC patients who underwent TA treatment. Especially, in one retrospective study from Yan et al with the largest sample sizes (414 cases), the overall incidence of local tumor progression rate after RFA was only $3.62 \%$ including LNM $(0.97 \%)$ and recurrence PTMC (2.42\%). Also, these patients who received additional RFA achieved good therapeutic results during follow-up. However, compared with PTMC, PTC (diameter > $10 \mathrm{~mm}, \mathrm{~T} 1 \mathrm{bN} 0 \mathrm{M} 0)$ patients who were enrolled to undergo the TA had a relatively higher residual lesion and LNM ratio (3.03\%, two in 66 cases; $1.52 \%$, one in 66 cases, respectively) in a recent study. Therefore, it's worth noticing that standardized management for TA patients is urgently needed, especially for patients who suffered from recurrence or LNM during the postoperative followup.

\section{Limitations and Prospect}

In the last few decades, significant progress has been made in the TA techniques for multiple early-stage cancers. The relatively long-term follow-up results in MWA, LA, and RFA were satisfactory that tumor VRR and complete absorption rate reached and even surpass 99\% (Figure $2 \mathrm{~A}$ ). For this reason, TA, as a new type of minimalinvasive treatment, seems to be a well-prepared alternative method for patients who have a strong desire for treatment but refuse or cannot tolerate surgery. There are still existing some limitations in widely applying these novel techniques. On the one hand, TA has been proposed for treating PTMC, triggering an extensive debate. Some scholars believed that it cannot only achieve the ultimate purpose of treatment of PTMC but further potentially help patients to relieve the psychological pressure on "cancerdiagnosis". Nowadays, AS is gradually accepted as a positive management option by clinicians and patients around the world. Noticeably, most recently, the Japan Association of Endocrine Surgery (JAES) updated the consensus statements about the indications and strategy for AS of low-risk PTMC patients, especially for the elderly population. ${ }^{62}$ Similarly, AS has been recommended to low-risk PTMC patients in the 2015 revised version guideline of the American thyroid association (ATA), ${ }^{63}$ and the cost-effective outcomes from the long-term follow-up have been recently determined. ${ }^{64,65}$ The follow-up examinations like cervical ultrasonography are mandatorily required once every six months in the first two years which can significantly avoid overlooking the need for surgery due to disease progression. Thus, some experts queried about the necessity of TA for PTMC in this new trend of the management for these kinds of inert cancers. However, one important problem during the AS that may easily be overlooked is the psychological uncertainty and anxiety could be stronger than immediate active interventions. ${ }^{4,66,67}$ Moreover, in the 2016 Chinese expert consensus $^{8}$ on this topic, the recommendation of close observation for low-risk PTMC patients was still controversial and had a more stringent criterion. Consequently, it seems that TA might be a good solution to compensate for image deriving PTMC overdiagnosis. ${ }^{68}$

On the other hand, in terms of research itself, there are still many gaps in the literature that will be crucial to fill. First and foremost, most clinical trials have relied on 
a single-center retrospective cohort study design that was inevitably vulnerable to recall bias. Second, available experimental data of the efficacy and safety of TA were mainly come from Asia, especially from China. Thus, it's still unclear whether these techniques can be also equally feasible among populations from other different races and regions. In the existing literature, the target patients who were enrolled to receive TA treatment were usually carried unifocal lesions. Besides, as deeply understanding the biological characteristics of PTMC, low-risk PTMC itself which is not combined with clinically apparent LNM has a favorable prognosis. ${ }^{11,64,69} \mathrm{Up}$ to now, however, there is still a lack of comparative study that aims to evaluate the long-term follow-up outcomes between TA, surgery, and AS. For this reason, to improve shared clinical decisionmaking and better treatment modality, clinicians should take care of the patients' complaints or self-reports during the follow-up assessment.

\section{Conclusion}

In general, this narrative review concludes that the efficacy and safety of TA have been preliminarily determined in the recently published literature. Compared with surgery, TA presents the same satisfactory therapeutic effects but minimal postoperative trauma which can significantly improve the patients' quality of life. Although significant progress has been made in the past few years on this topic, there are still many gaps we need to fill, including the comparative study between AS and TA intervention. Therefore, we expect future larger sample, multicenter, and prospective randomized controlled trials to validate the feasibility of TA in dealing with low-risk PTMC.

\section{Code Availability (Software Application or Custom Code)}

Not applicable.

\section{Data Sharing Statement}

The datasets generated during and/or analyzed during the current study are available from the corresponding author on reasonable request.

\section{Ethics Approval and Informed Consent}

Not applicable.

\section{Author Contributions}

All authors contributed to data analysis, drafting or revising the article, have agreed on the journal to which the article will be submitted, gave final approval of the version to be published, and agree to be accountable for all aspects of the work".

\section{Funding}

No funding was received to assist with the preparation of this manuscript.

\section{Disclosure}

The authors declare that they have no conflicts of interest.

\section{References}

1. Wang J, Yu F, Shang Y, Ping Z, Liu L. Thyroid cancer: incidence and mortality trends in China, 2005-2015. Endocrine. 2020;68 (1):163-173.

2. Bray F, Ferlay J, Soerjomataram I, Siegel RL, Torre LA, Jemal A. Global cancer statistics 2018: GLOBOCAN estimates of incidence and mortality worldwide for 36 cancers in 185 countries. CA Cancer J Clin. 2018;68(6):394-424. doi:10.3322/caac.21492

3. Kim J, Gosnell JE, Roman SA. Geographic influences in the global rise of thyroid cancer. Nat Rev Endocrinol. 2020;16(1):17-29. doi:10.1038/s41574-019-0263-x

4. Yoshida Y, Horiuchi K, Okamoto T. Patients' view on the management of papillary thyroid microcarcinoma: active surveillance or surgery. Thyroid. 2020;30(5):681-687. doi:10.1089/thy.2019.0420

5. Smulever A, Pitoia F. High rate incidence of post-surgical adverse events in patients with low-risk papillary thyroid cancer who did not accept active surveillance. Endocrine. 2020;69:587-595. doi:10.1007/s12020-020-02310-8

6. Al-Qurayshi Z, Nilubol N, Tufano RP, Kandil E. Wolf in sheep's clothing: papillary thyroid microcarcinoma in the US. $J$ Am Coll Surg. 2020;230(4):484-491. doi:10.1016/j.jamcollsurg.2019.12.036

7. Jeon MJ, Kim WG, Chung KW, Baek JH, Kim WB, Shong YK. Active surveillance of papillary thyroid microcarcinoma: where do we stand? Eur Thyroid J. 2019;8(6):298-306. doi:10.1159/ 000503064

8. Gao M, Ge M, Ji Q, et al. 2016 Chinese expert consensus and guidelines for the diagnosis and treatment of papillary thyroid microcarcinoma. Cancer Biol Med. 2017;14(3):203-211. doi:10.20892/j.issn.2095-3941.2017.0051

9. Meng K, Tian W, Lv Z, Song X. Horner's syndrome subsequent to minimally invasive video-assisted thyroidectomy in two patients. Oncol Lett. 2015;10(1):459-462. doi:10.3892/ol.2015.3159

10. Miyauchi A, Ito $\mathrm{Y}$, Oda $\mathrm{H}$. Insights into the management of papillary microcarcinoma of the thyroid. Thyroid. 2018;28(1):23-31. doi:10.1089/thy.2017.0227

11. Ito Y, Miyauchi A, Oda H. Low-risk papillary microcarcinoma of the thyroid: a review of active surveillance trials. Eur J Surg Oncol. 2018;44(3):307-315. doi:10.1016/j.ejso.2017.03.004

12. Zhang M, Tufano RP, Russell JO, et al. Ultrasound-guided radiofrequency ablation versus surgery for low-risk papillary thyroid microcarcinoma: results of over 5 years' follow-up. Thyroid. 2020;30(3):408-417. doi:10.1089/thy.2019.0147

13. Teng DK, Li WH, Du JR, Wang H, Yang DY, Wu XL. Effects of microwave ablation on papillary thyroid microcarcinoma: a five-year follow-up report. Thyroid. 2020. doi:10.1089/thy.2020.0049 
14. Trimboli P, Castellana M, Sconfienza LM, et al. Efficacy of thermal ablation in benign non-functioning solid thyroid nodule: a systematic review and meta-analysis. Endocrine. 2020;67(1):35-43. doi:10.1007/ s12020-019-02019-3

15. Papini E, Monpeyssen H, Frasoldati A, Hegedüs L. 2020 European Thyroid Association clinical practice guideline for the use of image-guided ablation in benign thyroid nodules. Eur Thyroid J. 2020;9(4):172-185.

16. Yue WW, Qi L, Wang DD, et al. US-guided microwave ablation of low-risk papillary thyroid microcarcinoma: longer-term results of a prospective study. J Clin Endocrinol Metab. 2020;105 (6):1791-1800. doi:10.1210/clinem/dgaa128

17. Yue W, Wang S, Yu S, Wang B. Ultrasound-guided percutaneous microwave ablation of solitary T1N0M0 papillary thyroid microcarcinoma: initial experience. Int J Hyperthermia. 2014;30(2):150-157. doi:10.3109/02656736.2014.885590

18. Zhou W, Ni X, Xu S, Zhang L, Chen Y, Zhan W. Ultrasound-guided laser ablation versus surgery for solitary papillary thyroid microcarcinoma: a retrospective study. Int $J$ Hyperthermia. 2019;36 (1):897-904. doi:10.1080/02656736.2019.1649475

19. Ding M, Tang X, Cui D, et al. Clinical outcomes of ultrasound-guided radiofrequency ablation for the treatment of primary papillary thyroid microcarcinoma. Clin Radiol. 2019;74 (9):712-717. doi:10.1016/j.crad.2019.05.012

20. Yan L, Lan Y, Xiao J, Lin L, Jiang B, Luo Y. Long-term outcomes of radiofrequency ablation for unifocal low-risk papillary thyroid microcarcinoma: a large cohort study of 414 patients. Eur Radiol. 2020. doi:10.1007/s00330-020-07128-6

21. Ji L, Wu Q, Gu J, et al. Ultrasound-guided percutaneous laser ablation for papillary thyroid microcarcinoma: a retrospective analysis of 37 patients. Cancer Imaging. 2019;19(1):16. doi:10.1186/s40644-019-0204-x

22. Papini E, Guglielmi R, Gharib H, et al. Ultrasound-guided laser ablation of incidental papillary thyroid microcarcinoma: a potential therapeutic approach in patients at surgical risk. Thyroid. 2011;21 (8):917-920. doi:10.1089/thy.2010.0447

23. Li J, Liu Y, Liu J, Qian L. Ultrasound-guided percutaneous microwave ablation versus surgery for papillary thyroid microcarcinoma. Int J Hyperthermia. 2018;34(5):653-659. doi:10.1080/ 02656736.2018 .1453092

24. Noguchi S, Yamashita H, Uchino S, Watanabe S. Papillary microcarcinoma. World J Surg. 2008;32(5):747-753. doi:10.1007/ s00268-007-9453-0

25. Tan HJ, Marks LS, Hoyt MA, et al. The relationship between intolerance of uncertainty and anxiety in men on active surveillance for prostate cancer. J Urol. 2016;195(6):1724-1730. doi:10.1016/j. juro.2016.01.108

26. Jeon MJ, Lee YM, Sung TY, et al. Quality of life in patients with papillary thyroid microcarcinoma managed by active surveillance or lobectomy: a cross-sectional study. Thyroid. 2019;29(7):956-962. doi:10.1089/thy.2018.0711

27. Tinguely P, Frehner L, Lachenmayer A, et al. Stereotactic image-guided microwave ablation for malignant liver tumors-a multivariable accuracy and efficacy analysis. Front Oncol. 2020;10:842. doi:10.3389/fonc.2020.00842

28. Iezzi R, Cioni R, Basile D, et al. Standardizing percutaneous Microwave Ablation in the treatment of Lung Tumors: a prospective multicenter trial (MALT study). Eur Radiol. 2020. doi:10.1007/s00330-020-07299-2

29. Filippiadis D, Mauri G, Marra P, Charalampopoulos G, Gennaro N, De Cobelli F. Percutaneous ablation techniques for renal cell carcinoma: current status and future trends. Int J Hyperthermia. 2019;36 (2):21-30. doi:10.1080/02656736.2019.1647352

30. Li X, Liang P, Yu J, et al. Role of contrast-enhanced ultrasound in evaluating the efficiency of ultrasound guided percutaneous microwave ablation in patients with renal cell carcinoma. Radiol Oncol. 2013;47(4):398-404. doi:10.2478/raon-2013-0038
31. Li J, Liu Y, Liu J, Yang P, Hu X, Qian L. A comparative study of short-term efficacy and safety for thyroid micropapillary carcinoma patients after microwave ablation or surgery. Int $J$ Hyperthermia. 2019;36(1):640-646. doi:10.1080/ 02656736.2019 .1626492

32. Vorländer C, David Kohlhase K, Korkusuz Y, et al. Comparison between microwave ablation and bipolar radiofrequency ablation in benign thyroid nodules: differences in energy transmission, duration of application and applied shots. Int J Hyperthermia. 2018;35 (1):216-225. doi:10.1080/02656736.2018.1489984

33. Liu F, Yu X, Liu Z, et al. Comparison of ultrasound-guided percutaneous microwave ablation and parathyroidectomy for primary hyperparathyroidism. Int $J$ Hyperthermia. 2019;36 (1):835-840.

34. Teng DK, Li HQ, Sui GQ, et al. Preliminary report of microwave ablation for the primary papillary thyroid microcarcinoma: a large-cohort of 185 patients feasibility study. Endocrine. 2019;64 (1):109-117. doi:10.1007/s12020-019-01868-2

35. Teng D, Sui G, Liu C, Wang Y, Xia Y, Wang H. Long-term efficacy of ultrasound-guided low power microwave ablation for the treatment of primary papillary thyroid microcarcinoma: a 3-year follow-up study. J Cancer Res Clin Oncol. 2018;144(4):771-779. doi:10.1007/s00432018-2607-7

36. Navarrete-Dechent C, Cordova M, Liopyris K, et al. Reflectance confocal microscopy-guided carbon dioxide laser ablation of low-risk basal cell carcinomas: a prospective study. J Am Acad Dermatol. 2019;81(4):984-988. doi:10.1016/j.jaad.2019.06.014

37. Sartori S, Mauri G, Tombesi P, Di Vece F, Bianchi L, Pacella CM. Ultrasound-guided percutaneous laser ablation is safe and effective in the treatment of small renal tumors in patients at increased bleeding risk. Int J Hyperthermia. 2018;35(1):19-25. doi:10.1080/02656 736.2018.1468038

38. Puls R, Langner S, Rosenberg C, et al. Laser ablation of liver metastases from colorectal cancer with MR thermometry: 5-year survival. J Vasc Interv Radiol. 2009;20(2):225-234. doi:10.1016/j. jvir.2008.10.018

39. Zhou W, Ni X, Xu S, Zhang L, Chen Y, Zhan W. Ultrasound-guided laser ablation versus microwave ablation for patients with unifocal papillary thyroid microcarcinoma: a retrospective study. Lasers Surg Med. 2020;52:855-862. doi:10.1002/1sm.23238

40. Jondal DE, Thompson SM, Butters KA, et al. Heat stress and hepatic laser thermal ablation induce hepatocellular carcinoma growth: role of PI3K/mTOR/AKT signaling. Radiology. 2018;288(3):730-738. doi:10.1148/radiol.2018172944

41. Naccarelli GV, Shih HT, Jalal S. Catheter ablation for the treatment of paroxysmal supraventricular tachycardia. $J$ Cardiovasc Electrophysiol. 1995;6(10 Pt 2):951-961. doi:10.1111/j.15408167.1995.tb00371.x

42. Huang SK. Advances in applications of radiofrequency current to catheter ablation therapy. Pacing Clin Electrophysiol. 1991;14 (1):28-42. doi:10.1111/j.1540-8159.1991.tb04044.x

43. Chu KF, Dupuy DE. Thermal ablation of tumours: biological mechanisms and advances in therapy. Nat Rev Cancer. 2014;14 (3):199-208.

44. Kim JH, Baek JH, Sung JY, et al. Radiofrequency ablation of low-risk small papillary thyroidcarcinoma: preliminary results for patients ineligible for surgery. Int $J$ Hyperthermia. 2017;33 (2):212-219. doi:10.1080/02656736.2016.1230893

45. Lim HK, Cho SJ, Baek JH, et al. US-guided radiofrequency ablation for low-risk papillary thyroid microcarcinoma: efficacy and safety in a large population. Korean J Radiol. 2019;20(12):1653-1661. doi:10.3348/ kjr.2019.0192

46. Zhang M, Luo Y, Zhang Y, Tang J. Efficacy and safety of ultrasound-guided radiofrequency ablation for treating low-risk papillary thyroid microcarcinoma: a prospective study. Thyroid. 2016;26 (11):1581-1587. doi:10.1089/thy.2015.0471 
47. Xiao J, Zhang M, Zhang Y, et al. Efficacy and safety of ultrasonography-guided radiofrequency ablation for the treatment of T1bN0M0 papillary thyroid carcinoma: a retrospective study. Int $J$ Hyperthermia. 2020;37(1):392-398. doi:10.1080/02656736. 2020.1752945

48. Wu R, Luo Y, Tang J, et al. Ultrasound-guided radiofrequency ablation for papillary thyroid microcarcinoma: a retrospective analysis of 198 patients. Int J Hyperthermia. 2020;37(1):168-174. doi:10.1080/ 02656736.2019 .1708480

49. Dong P, Wu XL, Sui GQ, et al. The efficacy and safety of microwave ablation versus lobectomy for the treatment of benign thyroid nodules greater than $4 \mathrm{~cm}$. Endocrine. 2020. doi:10.1007/s12020-020-02338-w

50. Jin H, Lin W, Lu L, Cui M. Conventional thyroidectomy versus thyroid thermal ablation on postoperative quality of life and satisfaction for patients with benign thyroid nodules. Eur J Endocrinol. 2020 .

51. Lan Y, Luo Y, Zhang M, et al. Quality of life in papillary thyroid microcarcinoma patients undergoing radiofrequency ablation or surgery: a comparative study. Front Endocrinol (Lausanne). 2020;11:249. doi:10.3389/fendo.2020.00249

52. Romero-Gómez B, Guerrero-Alonso P, Carmona-Torres JM, LaredoAguilera JA, Pozuelo-Carrascosa DP, Cobo-Cuenca AI. Sexual function in levothyroxine-treated hypothyroid women and women without hypothyroidism: a case-control. Int J Environ Res Public Health. 2020;17(12):4325. doi:10.3390/ijerph17124325

53. Wang Y, Wang H. Effects of hypothyroidism and subclinical hypothyroidism on sexual function: a meta-analysis of studies using the female sexual function index. Sex Med. 2020;8(2):156-167. doi:10.1016/j.esxm.2020.03.001

54. Oppo A, Franceschi E, Atzeni F, Taberlet A, Mariotti S. Effects of hyperthyroidism, hypothyroidism, and thyroid autoimmunity on female sexual function. J Endocrinol Invest. 2011;34(6):449-453. doi:10.1007/BF03346712

55. Zhang L, Zhou W, Zhan W, Peng Y, Jiang S, Xu S. Percutaneous laser ablation of unifocal papillary thyroid microcarcinoma: utility of conventional ultrasound and contrast-enhanced ultrasound in assessing local therapeutic response. World J Surg. 2018;42(8):2476-2484. doi:10.1007/s00268-018-4500-6

56. Xu B, Zhou NM, Cao WT, Gu SY. Comparative study on operative trauma between microwave ablation and surgical treatment for papillary thyroid microcarcinoma. World J Clin Cases. 2018;6 (15):936-943. doi:10.12998/wjcc.v6.i15.936

57. Chung SR, Baek JH, Choi YJ, Lee JH. Management strategy for nerve damage during radiofrequency ablation of thyroid nodules. Int J Hyperthermia. 2019;36(1):204-210. doi:10.1080/02656736. 2018.1554826

58. Ma B, Wei W, Xu W, et al. Surgical confirmation of incomplete treatment for primary papillary thyroid carcinoma by percutaneous thermal ablation: a retrospective case review and literature review. Thyroid. 2018;28(9):1134-1142. doi:10.1089/thy.2017.0558
59. Dodd GD, Dodd NA, Lanctot AC, Glueck DA. Effect of variation of portal venous blood flow on radiofrequency and microwave ablations in a blood-perfused bovine liver model. Radiology. 2013;267 (1):129-136. doi:10.1148/radiol.12120486

60. Du J, Li HL, Zhai B, Chang S, Li FH. Radiofrequency ablation for hepatocellular carcinoma: utility of conventional ultrasound and contrast-enhanced ultrasound in guiding and assessing early therapeutic response and short-term follow-up results. Ultrasound Med Biol. 2015;41(9):2400-2411. doi:10.1016/j.ultrasmedbio.2015.05.004

61. Meloni MF, Smolock A, Cantisani V, et al. Contrast enhanced ultrasound in the evaluation and percutaneous treatment of hepatic and renal tumors. Eur J Radiol. 2015;84(9):1666-1674. doi:10.1016/j. ejrad.2015.06.004

62. Sugitani I, Ito Y, Takeuchi D, et al. Indications and strategy for active surveillance of adult low-risk papillary thyroid microcarcinoma: consensus statements from the Japan Association of Endocrine Surgery Task Force on management for papillary thyroid microcarcinoma. Thyroid. 2020. doi:10.1089/thy.2020.0330

63. Haugen BR, Alexander EK, Bible KC, et al. 2015 American Thyroid Association Management guidelines for adult patients with thyroid nodules and differentiated thyroid cancer: the American Thyroid Association guidelines task force on thyroid nodules and differentiated thyroid cancer. Thyroid. 2016;26(1):1-133. doi:10.1089/ thy. 2015.0020

64. Sugitani I, Ito Y, Miyauchi A, Imai T, Suzuki S. Active surveillance versus immediate surgery: questionnaire survey on the current treatment strategy for adult patients with low-risk papillary thyroid microcarcinoma in Japan. Thyroid. 2019;29(11):1563-1571.

65. Cho SJ, Suh $\mathrm{CH}$, Baek JH, et al. Active surveillance for small papillary thyroid cancer: a systematic review and meta-analysis. Thyroid. 2019;29(10):1399-1408. doi:10.1089/thy.2019.0159

66. Hedman C, Djärv T, Strang P, Lundgren CI. Determinants of long-term quality of life in patients with differentiated thyroid carcinoma - a population-based cohort study in Sweden. Acta Oncol. 2016;55(3):365-369. doi:10.3109/0284186X.2015.1102965

67. Davies L, Roman BR, Fukushima M, Ito Y, Miyauchi A. Patient experience of thyroid cancer active surveillance in Japan. JAMA Otolaryngol Head Neck Surg. 2019;145(4):363-370.

68. Mauri G, Sconfienza LM. Image-guided thermal ablation might be a way to compensate for image deriving cancer overdiagnosis. Int J Hyperthermia. 2017;33(4):489-490. doi:10.1080/ 02656736.2016 .1262969

69. Ramundo V, Sponziello M, Falcone R, et al. Low-risk papillary thyroid microcarcinoma: optimal management toward a more conservative approach. J Surg Oncol. 2020;121(6):958-963. doi:10.1002/jso. 25848
Cancer Management and Research

\section{Publish your work in this journal}

Cancer Management and Research is an international, peer-reviewed open access journal focusing on cancer research and the optimal use of preventative and integrated treatment interventions to achieve improved outcomes, enhanced survival and quality of life for the cancer patient.
The manuscript management system is completely online and includes a very quick and fair peer-review system, which is all easy to use. Visit http://www.dovepress.com/testimonials.php to read real quotes from published authors. 